\title{
Available response choices affect localization of sound
}

\author{
STEPHEN PERRETT and WILLIAM NOBLE \\ University of New England, Armidale, Australia
}

\begin{abstract}
Successful replication of an experiment by Butler and Humanski (1992) showed that listeners are able to proficiently localize sources on a lateral vertical plane on the basis of interaural differences alone. When a lateral horizontal array was included in the test setup, that finding was replicated only for a broadband signal interacting with the pinna, not for ones (lowpass and pure tone) providing only interaural differences. Cross-plane errors conforming to "cones of confusion" were observed for those latter sounds. In a second experiment, response options were made more unconstrained, which clarified the nature of the cross-plane confusions. Lowpass signals from lateral vertical plane sources tend to be heard at or close to the horizon. Measurement of cue values needs to take account of the response options available to listeners, as well as signal properties.
\end{abstract}

Classical theory of sound localization in space has stressed the role of the binaural system. Early research (Stevens \& Newman, 1936) showed that the auditory system is sensitive to interaural time differences for lowfrequency sounds and interaural level differences for sounds of high frequency. The low-frequency cue is considered to be largely an effect of time and phase difference detection; the high-frequency cue is the result of increasingly sharp acoustic shadowing by the head. In classical theory, the head is assumed to be a perfect sphere, with the ears as holes located at the extremes of a diameter through the sphere (Mills, 1972). Using this model, interaural differences specify the horizontal angle of the sound source to the left or right of the median vertical plane (MVP). Absence of difference specifies that the source is somewhere on the MVP. Such differences cannot unambiguously specify the vertical angle of the sound source relative to the horizontal plane nor, relatedly, whether it lies forward or rearward of, or above or below, the interaural axis. In normal listening conditions, and with a broadband noise signal, those aspects of spatial whereabouts seemingly not revealed by "classical" interaural differences can nonetheless be distinguished.

In a recent report, Butler and Humanski (1992) presented results showing that listeners could accurately discriminate the whereabouts of sound sources displaced at $15^{\circ}$ intervals in a quadrant above the interaural axisin the lateral vertical plane (LVP). Proficient performance was observed for bursts of $3-\mathrm{kHz}$ highpass filtered noise. Such short wavelength signals interact with

\footnotetext{
The authors thank Bruce Stevenson for valuable advice about the design of Experiment 2. The research reported here was supported in part by grants from the Faculty Research Committee of the University of New England and from the Australian Research Council. Address correspondence to W. Noble, University of New England, Department of Psychology, Armidale, NSW 2351, Australia (e-mail: wnoble@ metz.une.edu.au).
}

the small cavities of the pinnae, creating alterations in the spectrum of the sound that vary systematically with change in vertical angle (Shaw, 1982). It has been assumed that these sorts of spectral effects enable identification of the whereabouts of acoustic events at different elevations relative to the horizon. Evidence supporting this is in the form of retained localization function for complex sounds displaced on the MVP (e.g., Hebrank \& Wright, 1974). In that plane, "classical" interaural differences are absent; the system relies on spectral changes that may be induced by each pinna, along with interaural spectral differences due to structural disparities between the two pinnae (Searle, Braida, Cuddy, \& Davis, 1975).

Butler and Humanski (1992) also found discrimination in the LVP for a $3-\mathrm{kHz}$ lowpass noise that approached the proficiency for highpass. The frequency content of such a signal would not be likely to interact with the pinnae. Those authors argued that the basis for this discrimination must be interaural differences, which also vary systematically with lateral elevation change (Mills, 1972). As corroboration of that argument, it was observed that the lowpass signal (1) was not distinguished regarding whereabouts on the LVP when heard monaurally, and (2) was not distinguished above $30^{\circ}$ elevation when heard binaurally in the MVP. These outcomes suggest that the signal did not contain a material component productive of pinna/e-induced spectral cues; hence, its accurate localization was indeed largely due to the discrimination of interaural differences in time/phase and level.

The loudspeaker array in Butler and Humanski's experiment was confined to a vertical quadrant. The LVP in their case described a quadrant, any point on which lay on a "cone of confusion" (Woodworth, 1938), such that an interaural difference in time or level could, in theory, specify a source $n^{\circ}$ above the interaural axis or $n^{\circ}$ below, or $n^{\circ}$ forward or rearward of it, or at any equivalent position intermediate between lateral vertical and horizontal planes. Thus, proficient LVP performance for 
the lowpass signal could have arisen, not because listeners were making "vertical plane discriminations," but because they were "discriminating interaural differences" and were constrained in the available locations to which they could attribute those differences.

A feature of the results from two recent experiments (Noble, Byrne, \& LePage, 1994; Noble, Murray, \& Waugh, 1990) led us to hypothesize that a factor of this kind could have operated. Both of these experiments involved simultaneous testing of localization in the lateral vertical and lateral horizontal planes, using broadband ("pink") noise signals and intersecting hemicircumferential arrays of loudspeakers, $18^{\circ}$ apart, spanning both planes. In one experiment, performance under normal conditions was compared with that when listeners wore different types of standard ear protectors. These have the twofold effect of obstructing the filtering potential of the pinnae, while also acting as lowpass filters. A consistent finding under ear protector listening was the notable flight in attributions of location from LVP positions to ones at or near "cone-of-confusion" equivalents on the lateral horizontal plane (LHP). The opposite migration occurred much less frequently. In the other experiment, the same loudspeaker and signal arrangement was used to compare the localization function of normally hearing listeners with that of listeners with high-frequency hearing loss - again the equivalent of lowpass filtering. The phenomenon of misattribution of broadband signals on the LVP to exact or approximate cone-of-confusion positions on the LHP was observed among (only) the listeners with impaired hearing. A detail of Butler and Humanski's data (1992, p. 185) also suggests that available response choices affected their results. When highpass sounds were presented at $90^{\circ}$ elevation in the MVP, they were heard as located, on average, at $75^{\circ}$ elevation. When such sounds were presented at the identical location but in the context of the LVP, they were heard as located at $90^{\circ}$.

We therefore devised Experiment 1 to compare outcomes from a loudspeaker arrangement that replicates the response choices of Butler and Humanski's (1992) experiment with an arrangement in which response choices were available in the lateral horizontal as well as lateral vertical planes. We used signals replete with as well as ones devoid of energy affected by the pinna.

\section{EXPERIMENT 1}

\section{Method}

Twelve people participated as listeners ( 9 females, 3 males); all reported normal hearing function. They listened to signals generated from loudspeakers (Realistic Midrange Tweeters) selected from a larger set as having similar frequency responses to each other. These were mounted on lengths of 2-cm-section box pipe, bent to form circular segments. The loudspeakers were arranged in two configurations, one of which exactly replicated the layout in Butler and Humanski's experiment-that is, seven loudspeakers in a quadrant of $1.2 \mathrm{~m}$ radius, spanning vertically upward from the horizon $\left(0^{\circ}\right)$ at $15^{\circ}$ intervals to $+90^{\circ}$. The other configuration added six further matching loudspeakers arranged $15^{\circ}$ apart on a $1.2-\mathrm{m}$ radius horizontal quadrant, which ran counterclockwise from the base of the vertical arc and could be detached from it. The loudspeaker at $0^{\circ}$ of elevation was common to both the vertical and the horizontal quadrants. The apparatus was housed in a sound-isolated, semianechoic chamber, $3 \mathrm{~m}$ wide $\times 3.5 \mathrm{~m}$ long $\times 3 \mathrm{~m}$ high.

Testing was conducted using both configurations, with counterbalancing of test order in such a way that half the listeners encountered the vertical-only ( $\mathrm{Vo}$ ) array on an initial test, the verticalhorizontal (VH) one on the occasion of a repeat test. The opposite order was used for the other 6 participants. Testing in the vertical plane was confined to the lateral vertical, with the listeners tested in two orientations: LVP opposite the left ear, and LVP opposite the right ear (interaural axis aligned with the loudspeaker at $0^{\circ}$ elevation). This meant that in the VH condition, the leftward LVP was accompanied by a rear horizontal quadrant, and the rightward LVP by a front horizontal quadrant. The two orientations were used to control for any effect of a visible versus nonvisible horizontal array. All conditions were counterbalanced across listeners.

Signals were single $800-\mathrm{msec}$ bursts with $100-\mathrm{msec}$ rise and fall times delivered at a mean level of $60 \mathrm{dBA}$. This was randomly altered, trial by trial, by 0 or $\pm 2 \mathrm{~dB}$ to prevent the listeners from using minor level differences between loudspeaker positions as a possible cue with which to identify their locations. Three different signals were employed: broadband ("pink") noise (equal energy per $1 / 3$ octave from $250 \mathrm{~Hz}$ to $12.5 \mathrm{kHz}$ ), $400-\mathrm{Hz}$ lowpass noise (with $18 \mathrm{~dB}$ per octave rejection slope), and $400-\mathrm{Hz}$ pure tone. The broadband signal contains all the cues found in a highpass one; the lowpass was designed to offer only interaural difference cues. The signals used for our first experiment are analogous to those used by Butler and Humanski, but do not match them exactly; at that stage our filter system was not steep enough to ensure confidence that a higher frequency cutoff lowpass noise would be devoid of energy affected by the pinna. The tonal signal was used to check that the lowpass signal was indeed offering no such cue. The order of signal types was counterbalanced across listeners and test sessions.

A trial involved the presentation of a sound burst followed by the listeners verbally identifying the number of the loudspeaker they thought the sound had come from. In any block of trials, only one of the three signal types was used. There were 14 trials per block in sessions involving the Vo setup (each loudspeaker activated twice in a random order) and 13 trials per block in sessions involving the VH setup (each loudspeaker activated once in a random order). Each test session involved six blocks of trials ( 3 signal types $\times 2$ orientations). The listeners were seated with the center of the head at the center of the sphere implied by the loudspeaker layout. To allow responses without head movement, a miniature replica of the particular setup being used was placed in front of the listener. The loudspeaker locations of the replicas were numbered in the same way as those of the full-sized setups. Monitoring by CCTV ensured that no movement occurred during trials. An instruction to remain immobile has been found to be effective for maintenance of that posture (Noble, 1981). No training or feedback was given.

Data for the Vo setup (replication of Butler and Humanski's) were analyzed in two ways: average perceived location for each loudspeaker position and average error score across positions. An error score was calculated as the difference in number of loudspeakers between actual and nominated position. This value can be expressed both as average loudspeaker deviation and as deviation in degrees of arc. Data for the VH setup are not all meaningfully expressible in these terms. We may analyze the average withinplane errors in the foregoing way. Of greater relevance is the proportion of responses occurring as cross-plane misattributions and, more pertinent again, the nature of those cross-plane errors- that is, whether or not they conform to cone-of-confusion principles. In 
calculating those values, trials with the loudspeaker at $0^{\circ}$ of elevation common to both planes were excluded.

\section{Results}

Data were analyzed first of all to check for any test order effects. There were some nonsignificant trends due to that factor, but none strong enough to warrant separate presentation of results from different orders of orientation, configuration, or signal.

Lateral vertical plane only. Bar graphs in Figure 1 show the average error scores (and standard errors) in the Vo setup for the three signal types. Shown alongside these are equivalent results reported by Butler and $\mathrm{Hu}$ manski. Our data show that all the responses were generally accurate, somewhat more so for a broadband signal than for a lowpass or tone. The difference between broadband and lowpass noise is small in geometric terms (but statistically significant, $p<.01$ ). The difference between lowpass and tone is neither geometrically noticeable nor statistically significant. The absence of a noticeable difference between lowpass noise and tone suggests that the filtered noise did not provide cues beyond interaural (largely time/phase) differences. Our results for broadband and lowpass signals look quite similar to Butler and Humanski's for high- and lowpass (the difference in their case was also statistically significant).

In Figure 2 we plot the average perceived locations for the three types of signal for the Vo array. This confirms the finding of general accuracy for all three types of sound and replicates the result observed by Butler and Humanski. Our listeners proficiently discriminated the locations of signals in the lateral vertical plane whether or not those sounds contained information other than that arising from interaural time and level differences.

This experiment was not designed as a complete replication, and it is useful to note a feature of data from a different experiment, which conforms to another finding

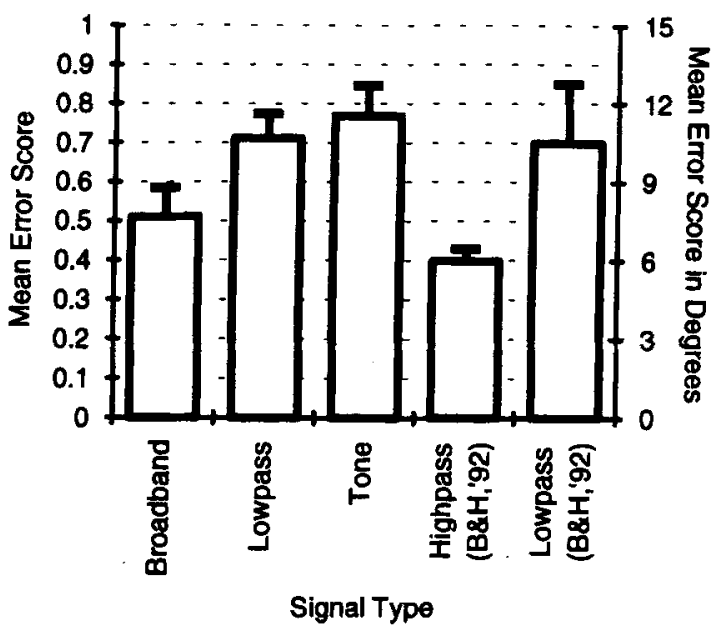

Figure 1. Mean error scores $(+S E)$ for broadband, lowpass, and tone presented in the lateral vertical plane, and values for highpass and lowpass obtained by Butler and Humanski (1992).

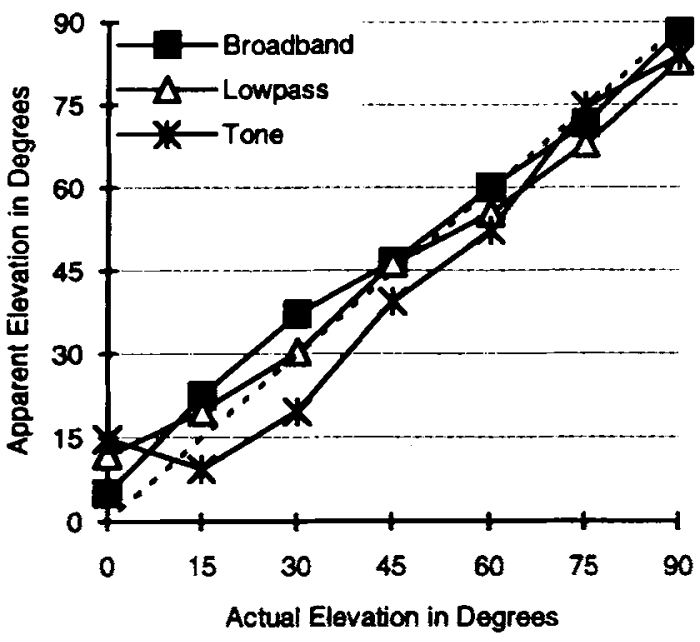

Figure 2. Apparent elevation of lateral vertical plane sources plotted against actual elevation, for broadband, lowpass, and tonal sounds.

by Butler and Humanski. In a revised test setup (to be described in the next section), we compared performance for broadband and lowpass signals in the median vertical plane. We obtained mean error scores of 1.21 and 2.67 for broadband and lowpass signals, respectively; Butler and Humanski obtained values of 0.53 and 1.73. Figure 3 shows the distinctions found in both experiments between the two signal types in the median vertical plane (as well as some differences in outcome across the two experiments). This is a spatial region providing only pinna/e-generated information for locale. All in all, it is clear that essential features of the previous experiment were corroborated in our laboratory. Conditions between our revised setup and Butler and Humanski's test in the MVP were sufficiently different so that we would not expect a close agreement in resultsa matter explicated in the Discussion. Incidentally, our result for the broadband signal at $90^{\circ}$ elevation in the MVP (Figure 3) differs from that at $90^{\circ}$ in the LVP (Figure 2). These are identical locations, underscoring the "context" effect found by Butler and Humanski.

Lateral vertical and horizontal planes. When listeners are presented with broadband, lowpass, and tonal signals from both the LVP and LHP (VH setup), profoundly different outcomes occur. Performance remains highly accurate for a broadband signal. Listeners correctly identify both azimuth and elevation (plane) on almost every trial. For those lowpass and tonal signals attributed to the plane of their origin, accuracy is similar to that found in the Vo condition. Such planar attribution is not guaranteed, however. As shown in Figure 4, 36.9\% of trials for the lowpass signal produced attributions to the other plane. Of those cross-plane errors, $45 \%$ ( $16.8 \%$ of trials) were in the form of attributions to equivalent positions on the other plane (exact cone-of-confusion errors); a further $39 \%$ were to positions immediately adjacent, giving a total of $84 \%$ exact and "fuzzy" cone-of-confusion 


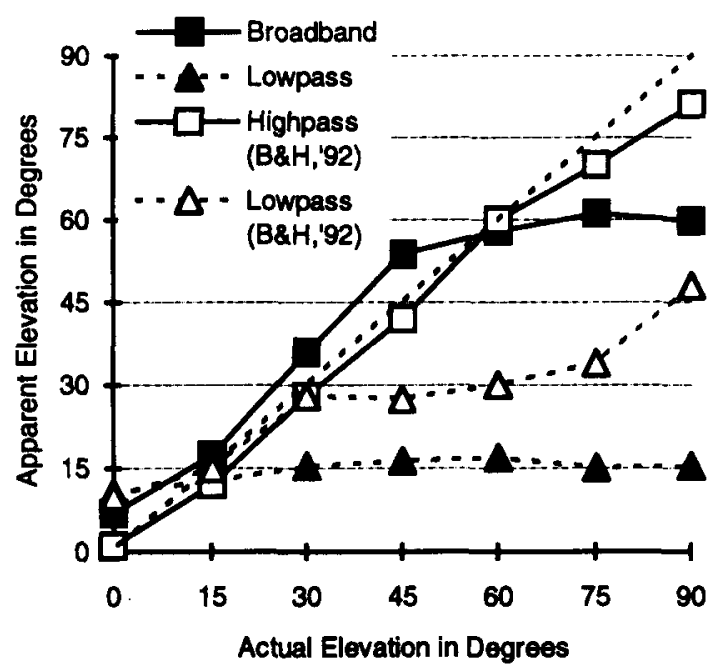

Figure 3. Apparent elevation of median vertical plane sources plotted against actual elevation, for broadband and lowpass sounds, with equivalent high- and lowpass results from Butler and Humanski (1992).

errors ( $31.1 \%$ of trials). The inclusion of "fuzzy" coneof-confusion errors is legitimate in view of imperfections and individual differences in the mapping of spatial geometry to measured interaural differences (e.g., Wightman \& Kistler, 1993). There were similar proportions of cross-plane errors occurring as either exact or fuzzy coneof-confusion responses for lowpass signals originating in the LVP $(39.2 \%)$ and in the LHP (44.3\%).

The extent of cross-plane attributions is greater for the tonal signal than for the lowpass noise, with a similar proportion of such attributions ( $81 \%$ ) occurring as exact or fuzzy cone-of-confusion errors. The contrast in outcomes

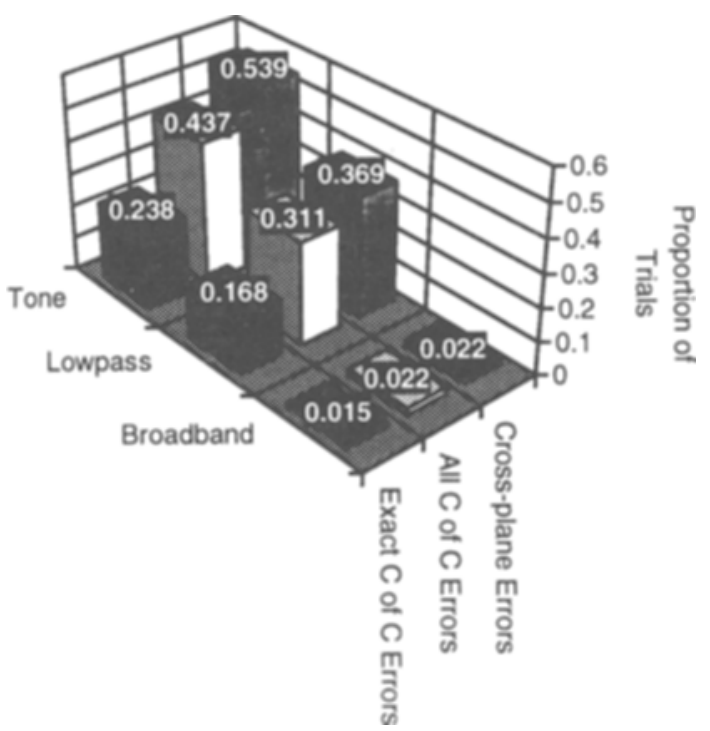

Figure 4. Proportions of cross-plane errors, all cone-of-confusion errors, and exact cone-of-confusion errors, for tone, lowpass, and broadband signals. between either of those signals and the broadband noise is the critical outcome. If the listeners were quite unable to determine the plane on which a sound arises, we would expect the cross-plane error rate to be around $50 \%$. The rate for the tone $(53.9 \%)$ indicates just such a chance level of performance. The rate for the lowpass noise (36.9\%) suggests the presence of a cue serving to bias responses to a small extent to the plane of origin. The overall result is quite clear. In contrast to the task parameters when only one quadrant is available for attribution of interaural differences, once listeners must determine between planes as well as among azimuths, they cannot rely on interaural differences alone to make accurate judgments.

The foregoing outcome demonstrates how variations in available response choice options may critically affect the pattern of responses observed. A related point has been noted before; Searle, Braida, Davis, and Colburn (1976) modeled the effect of changes in the area spanned by arrays of loudspeakers (hence, the response choices available) on the localization error to be expected. One outcome that underscores this point and raises further questions is the differential effect of the two different orientations on cross-plane errors. With the LVP to the listener's left (and the horizontal quadrant rearward of it), there were about the same number of vertical-tohorizontal cross-plane errors $(32 \%)$ as there were horizontal to vertical (28\%). With the LVP to the right (and the horizontal quadrant forward of it), vertical-tohorizontal misattributions were $35 \%$, but horizontal-tovertical error was significantly greater at $52 \%$. Given the constraints of our test setup, it is not possible to know whether listeners were actually making "cross-plane" errors, or were making errors that would be revealed as front-rear confusions if response options were to be even less constrained. Freeing up response options would also clarify the extent to which an elevation cue is present, even in a lowpass signal.

It is evident that more real assessments of localization function under various signal or listening conditions would be yielded if response options were unconstrained. This is by no means a novel observation, and investigators have adopted various strategies to try to achieve less constrained conditions. For example, Thurlow and Runge (1967) used blindfolding and had listeners aim a pointer at perceived locations; Oldfield and Parker (1984) used a similar procedure. Wightman and Kistler (1989) used blindfolding and had stationary listeners verbally identify perceived locations using spherical coordinates. Makous and Middlebrooks (1990) had listeners, in darkness, turn to face the perceived origin of a sound. They used an electromagnetic sensing device, with one component attached to the listener's head, to identify the direction of each "facial point."

\section{EXPERIMENT 2}

\section{Method}

We consider that a response involving the listeners' reorienting to face where they heard the sound coming from has the most ev- 


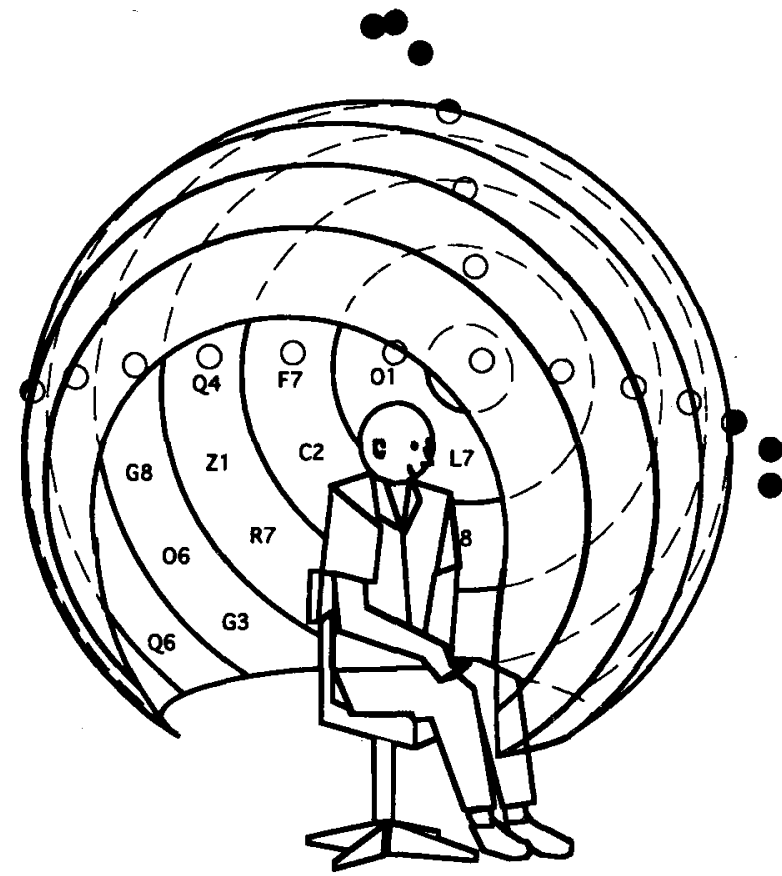

Figure 5. Loudspeaker layout relative to partial sphere structure. A sample area of the coordinate marking of the interior surface is shown. Coordinate labels use irregular two-term letter-number strings; coordinate positions occur at $15^{\circ}$ intervals of azimuth and elevation.

ident ecological validity. We also favor normal visibility and unencumbered listening. Hence, our strategy was to visually mask the whereabouts of actual loudspeaker positions and have listeners verbally report locations as identifiable from coordinates marked on the interior of an otherwise blank spherical surface. A further array of six matching loudspeakers was added to the apparatus to complete the horizontal hemicircumference and thus enable scrutiny of relations among the LVP and LHP.

The listeners were 18 people ( 10 females, 8 males) with reported normal hearing. They heard signals from a total of 19 loudspeakers -7 spanning the left lateral vertical plane $\left(0^{\circ}\right.$ to $+90^{\circ} \mathrm{el}-$ evation) and 12 spanning the left lateral horizontal plane $\left(0^{\circ}\right.$ to $180^{\circ}$ azimuth, where $0^{\circ}$ azimuth $=$ front-facing position); one loudspeaker (at $270^{\circ}$ azimuth and $0^{\circ}$ elevation) was common to both planes. To achieve an unconstrained response space, actual loudspeaker positions were visually masked. A skeletal spherical form, with a $1.0-\mathrm{m}$ radius, was constructed using $1-\mathrm{cm}$-diam metal rods, bent into hoops. Inside this structure was attached a fine-weave, acoustically transparent cloth whose surface was mapped, using an irregular coordinate system, as shown in part in Figure 5. Except for the area left open to enable the listeners to access and sit inside this construction, response options were available over a considerable portion of the two-dimensional spherical space. The sphere was positioned so that, as projected from its center, the loudspeaker array was exactly aligned with a subset of the coordinates. The loudspeakers were not in contact with the structure, and none were impeded by it. Lighting conditions were arranged so as to make it impossible to see any feature of the test room beyond the surface of the cloth.

As the procedure entailed listener reorientation from a standard straight-ahead position, yet we did not want movement-induced transformations confounding the measurement, it was determined that each signal be $150 \mathrm{msec}$ in duration $(10-\mathrm{msec}$ rise and fall times). This was observed, from scrutiny of video recordings, to be brief enough to come in under the movement reaction time of every listener on virtually every trial - an observation in agreement with that of Makous and Middlebrooks (1990). There were two signal types-broadband and $2-\mathrm{kHz}$ lowpass noise; the latter was shaped by a custom-built digital filter affording a rejection slope in excess of $72 \mathrm{~dB} /$ octave. The choice of 2 rather than $3 \mathrm{kHz}$, as used by Butler and Humanski, was to ensure no likelihood of pinna cues; those authors reported that 1 of their 6 listeners could discriminate in the MVP with a $3-\mathrm{kHz}$ but not a $2-\mathrm{kHz}$ lowpass signal. Our listeners underwent four blocks of 38 trials each (two activations of each loudspeaker per block, in random order), alternating the two signal types from one block to the next, half the sample starting with broadband noise. Other details of signal conditions are as those described for Experiment 1.

\section{Results}

Our interest was, first, to determine the extent to which elevation cues are exhibited in the lateral vertical plane, so as to clarify the cue value in different signals when response choice is less constrained. To observe this, we measured the extent to which the elevations of signal presentations originating in each of the three quadrantshorizontal plane forward and rearward of the listener, lateral vertical-gave rise to correlated elevation responses. Second, we wished to observe the extent to which confusion among the horizontal quadrants takes the form of front/rear reversal, so as to clarify the status of the cross-plane confusions observed in the first experiment.

Elevation cues. Figure 6 shows the average elevation component of responses to the broadband signal in each of the three quadrants, along with the average for the highpass noise used by Butler and Humanski. The ab-

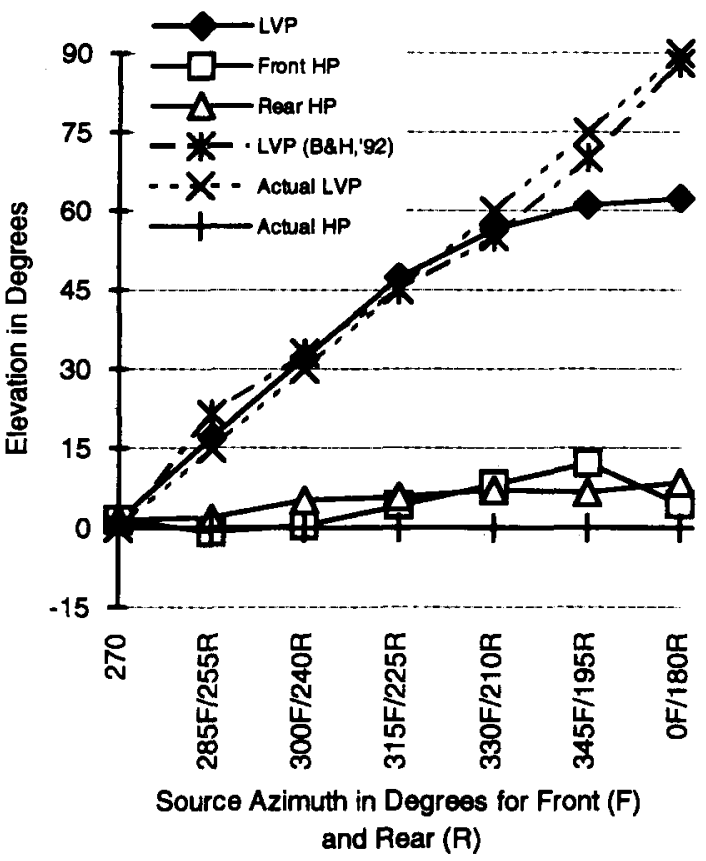

Figure 6. Elevation component in responses to broadband sounds presented from the lateral vertical plane (LVP), front and rear horizontal plane (HP), with equivalent results for highpass sound from Butler and Humanski (1992). 
scissa gives source positions as derived from the doublepole coordinate system of Middlebrooks, Makous, and Green (1989). They are thus expressed in terms of cones of confusion, separated by $15^{\circ}$ intervals. It is clear that, except for a deviation above $60^{\circ}$, our LVP data are in accord with those of Butler and Humanski, showing that discrimination remains proficient even when response choice is relatively unconstrained. There is also no noticeable departure in the perceived location of a broadband signal, activated at the horizon, from that $0^{\circ}$ elevation. Figure 7 shows the average elevation component of responses to the $2-\mathrm{kHz}$ lowpass signal and the result for Butler and Humanski's lowpass. The contrast with their finding (and with our own from Experiment 1 , under response conditions replicating theirs) is very clear. When response choice is unconstrained, lowpass signals presented in the LVP show only a modest degree of discriminability regarding their elevation, to about $30^{\circ}$; thereafter, they appear to originate increasingly from the horizon. There is an apparent, slight mimicking of this effect for signals presented in the forward horizontal quadrant.

Cross-plane and front/rear confusions. Providing an accessible representation of source-response relationships in spherical two-dimensional space is not easy (one advantage of constrained response choices is reduced data handling problems). The relative lack of constraint on responses frees listeners to identify apparent locations at intermediate points and at points outside the limits of the loudspeaker array. Thus, there are no neat ways to categorize responses in terms of types of confusion. The results shown in Figure 7, combined with our inspection of

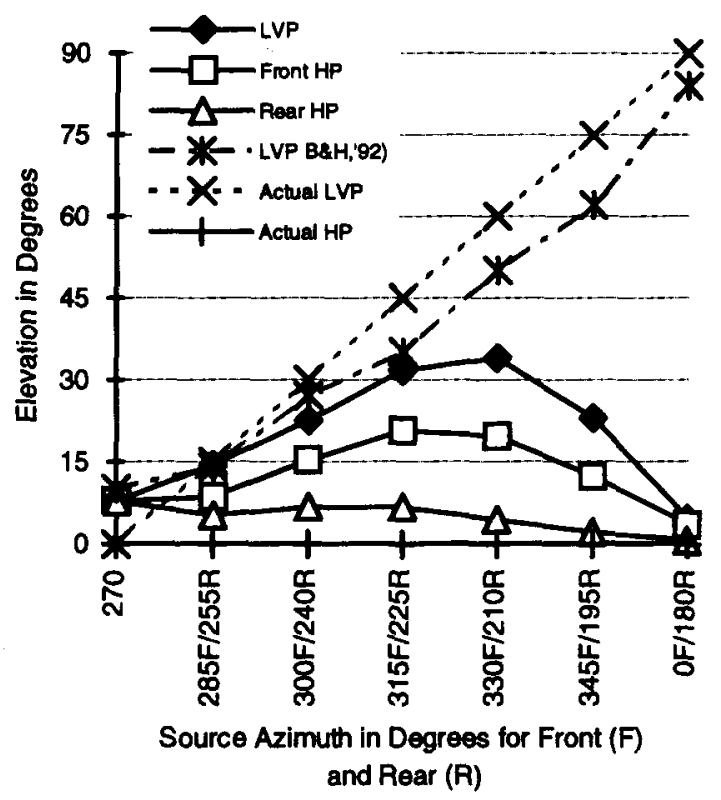

Figure 7. Elevation component in responses to lowpass sounds presented from the lateral vertical plane (LVP), front and rear horizontal plane (HP), with equivalent results for lowpass sound from Butler and Humanski (1992).

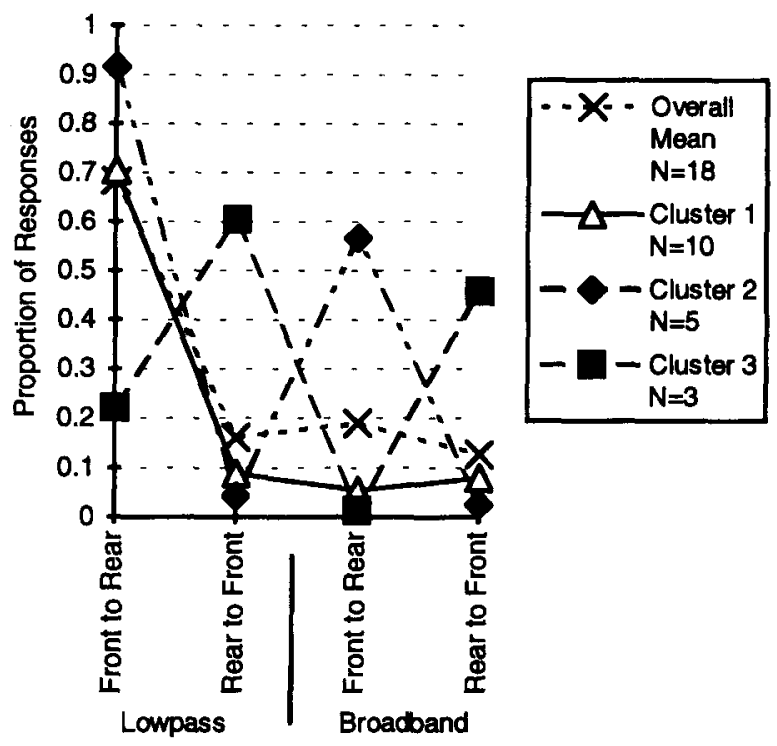

Figure 8. Cluster analysis showing proportions of different types of response for the whole sample and for three distinct cluster groups of listeners.

individual source-response scatterplots, make it clear that a lowpass signal does not enable substantial departures from the horizon, even for signals originating in the LVP. This is most evident at the upper extreme of that plane, where most responses are to cone-of-confusion equivalent positions in the horizontal plane.

A cluster analysis on front/rear confusions (Figure 8 ) showed that a high proportion of confusions for the lowpass signal are in the form of frontward sources heard as rearward. A cluster of 10 listeners show this front-to-rear bias strongly, with very little of the opposite confusion, nor of such error for the broadband signal. A second cluster $(n=5)$ showed the front-to-rear bias for both types of signal; a third $(n=3)$ showed the opposite bias for both types of signal. In Figures 9 and 10 we display the actual lowpass and broadband source-response relationships for 1 listener who is representative of the largest cluster group. From these it may be seen that the lowpass front-to-rear confusion has an elevation component apparent for signals off the median plane. The appearance of an elevation component in front HP responses (Figure 7) is thus due to cone-of-confusion attributions rearward that do not quite sit at the horizon.

These findings allow a conclusion that the appearance of front HP-to-LVP "cross-plane" errors in Experiment 1 was in fact the constrained attribution to the only loci "rearward" of the front HP, and vice versa for the rear HP-to-LVP. The listeners would have otherwise attributed those signals to rearward or frontward HP (or close to HP) locations. The LVP-to-HP migrations were thus not actually matched by confusions of the opposite kind, a finding consistent with those reported by Noble et al. (1994, Noble et al., 1990). 


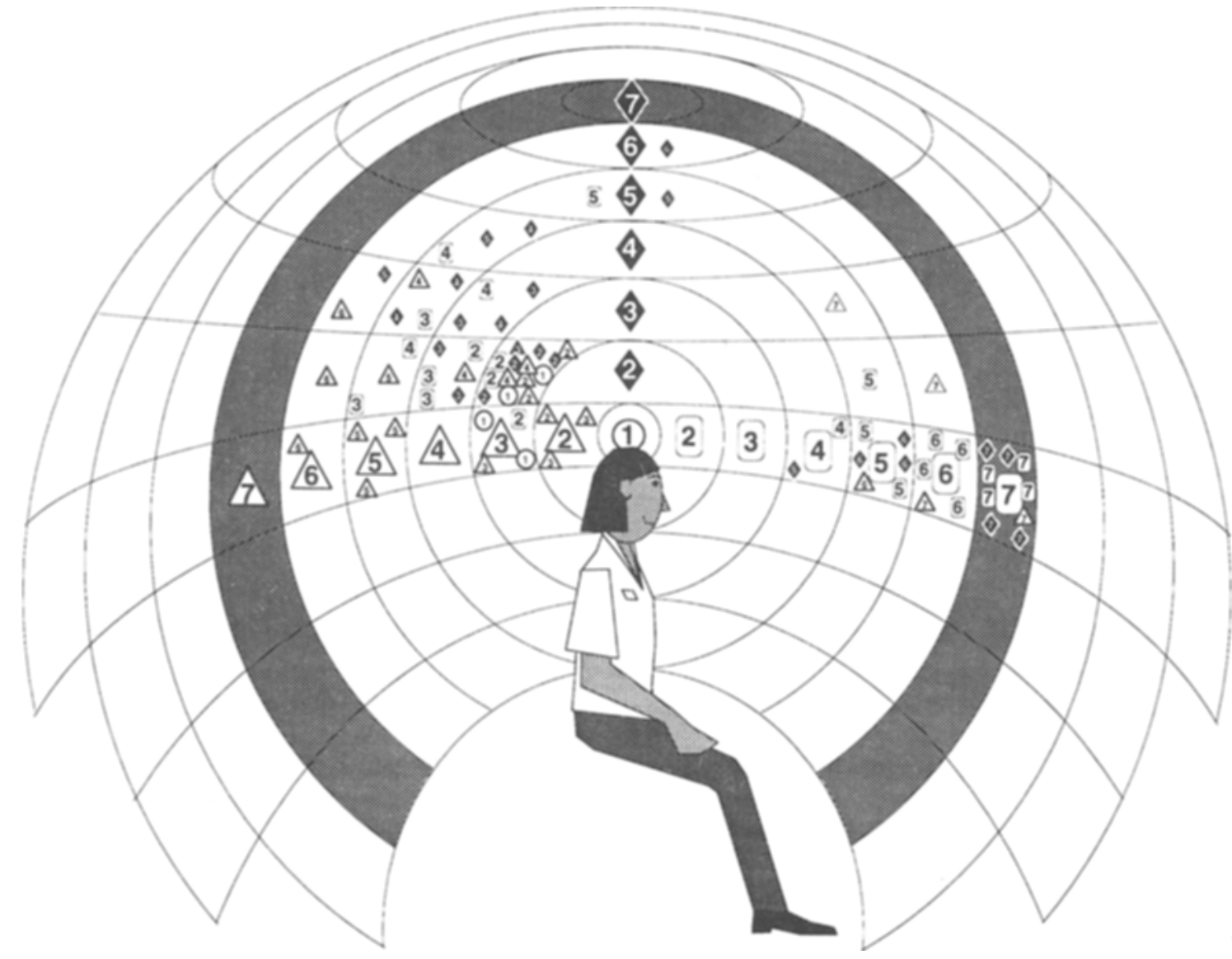

Figure 9. Scatterplot of source-response relations for a single listener from the largest cluster group, lowpass sound. Large logos show actual loudspeaker positions; small logos show response positions. Shaded band represents MVP. Area inside shaded band represents left hemifield; area outside shaded band represents right hemifield folded out beyond MVP.

\section{DISCUSSION}

These experiments demonstrate that altering the constraints on response choices can have critical influences on the measurements obtained in localization tasks. Thus, in addition to considerations of signal and listening parameters, for assessment of cue values one needs to take account of the degrees of freedom available for listeners' responses. We believe that this factor accounts for the major differences between the present and a previous study. It may also account for divergences between present and previously reported results, even in conditions where we also see replication. For instance, with our revised test setup, in both MVP and LVP, for a broadband signal we observed similar proficiency up to about $60^{\circ}$ to that found by Butler and Humanski with their setup, but we did not observe such proficient performance at the upper extreme of those planes (and we did get results identical to theirs in the LVP by using a replica of their setup).

For the LVP, the explanation for the difference may be seen in Figure 10. Responses become more wayward in the uppermost part of the LVP in terms of elevation. This is calculated as "downward" elevation error, thus affect- ing the plot (Figure 6) of average perceived location. In an experiment like that of Butler and Humanski, where the "vertical" location of each source can be determined in terms of azimuth, because responses cannot stray to other, less elevated regions of the cone-of-confusion, performance appears proficient throughout the plane. In the MVP, we observed an increasing tendency for responses to "overshoot" to positions rearward of the zenith with sources elevated above $45^{\circ}$. Again, these count as downward elevation errors and affect the plot similarly (Figure 3). With locations bounded at the zenith, this sort of error cannot manifest itself.

The results reported here do not support an argument for diminishing the role of interaural differences in vertical plane discrimination, nor was it assumed that they would. There is evidence that interaural differences are involved in enabling elevation as well as azimuth to be discriminated, for any source outside the MVP. Butler, Humanski, and Musicant (1990) found more accurate binaural than monaural localization at regions outside the MVP, especially in the upper area of the hemifield. Makous and Middlebrooks (1990), Butler et al. and Noble et al. (1994) reported error magnitudes that were lower in LVP than MVP judgments. 


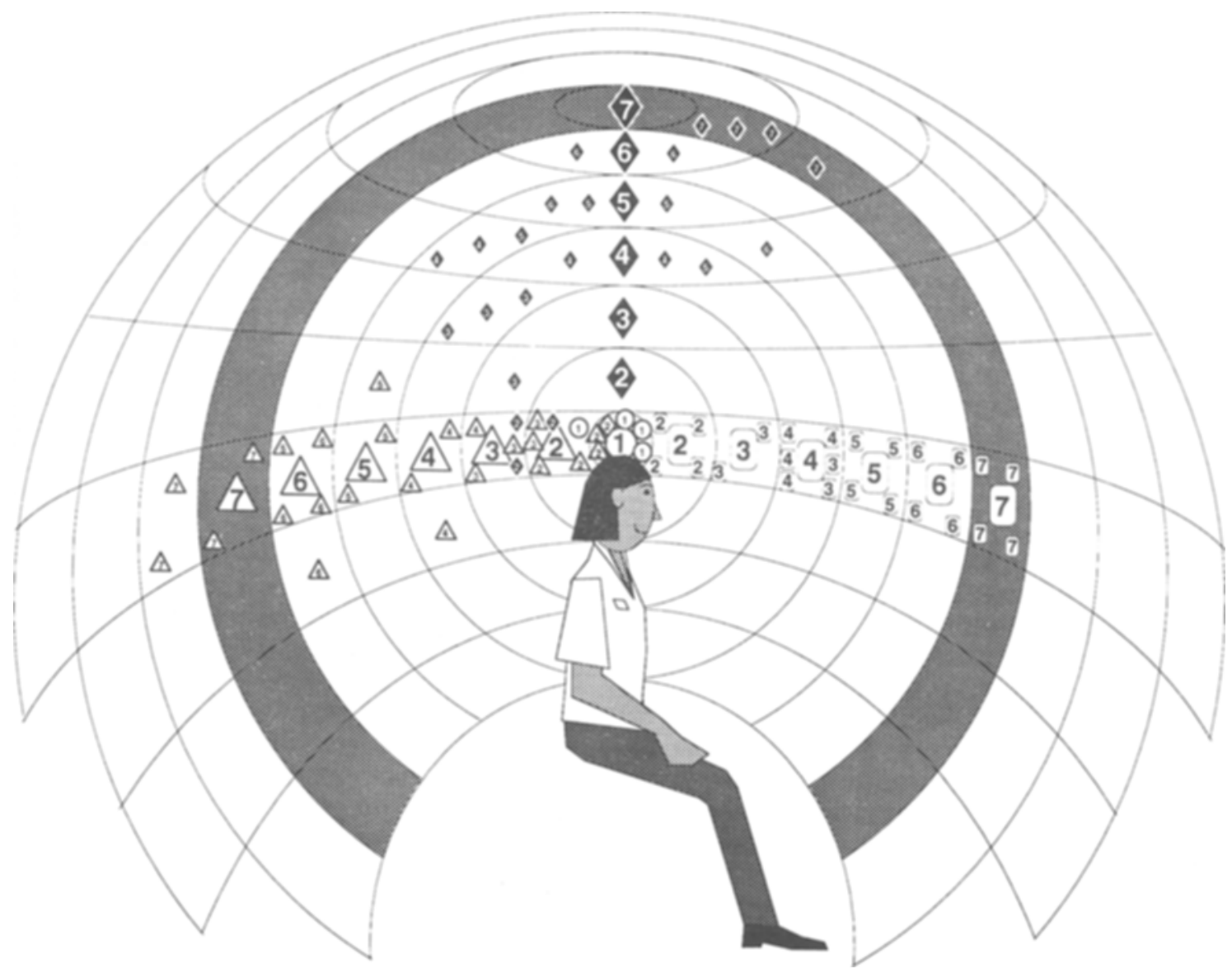

Figure 10. Scatterplot for a listener as in Figure 9, broadband sound.

Our data suggest how the auditory system "weighs" input. Other things being equal, when faced with a signal that combines pinna-derived elevation cues and interaural differences specifying azimuth, locations are perceived to be at or close to their real coordinates. Faced with a signal in which a clear elevation cue is absent, the typical default is toward the horizon, presumably because interaural differences dominate in the specification of horizontal displacements; hence, prior likelihoods favor that region of two-dimensional space. There are indications in the data of some lateral elevation discrimination, even for a signal that is unlikely to offer any component for interaction with the ipsilateral pinna. This may relate to effects of the torso (Gardner, 1973; Kuhn, 1987), a question that is the subject of continuing investigation.

\section{REFERENCES}

Butler, R. A., \& Humanski, R. A. (1992). Localization of sound in the vertical plane with and without high-frequency spectral cues. Perception \& Psychophysics, 51, 182-186.

Butler, R. A., Humanski, R. A., \& Musicant, A. D. (1990). Binaural and monaural localization of sound in two-dimensional space. Perception, 19, 241-256.

GARDNER, M. B. (1973). Some monaural and binaural facets of median plane localization. Journal of the Acoustical Society of America, 54, 1489-1495.

Hebrank, J., \& Wright, D. (1974). Are two ears necessary for localization of sound sources on the median plane? Journal of the Acoustical Society of America, 56, 935-938.

KUHN, G. F. (1987). Physical acoustics and measurements pertaining to directional hearing. In W. A. Yost \& G. Gourevitch (Eds.), Directional hearing (pp. 3-25). New York: Springer-Verlag.

Makous, J. C., \& Middlebrooks, J. C. (1990). Two-dimensional sound localization by human listeners. Journal of the Acoustical Society of America, 87, 2188-2200.

Middlebrooks, J. C., Makous, J. C., \& Green, D. M. (1989). Directional sensitivity of sound-pressure levels in the human ear canal. Journal of the Acoustical Society of America, 86, 89-108.

Mills, A. W. (1972). Auditory localization. In J. V. Tobias (Ed.), Foundations of modern auditory theory (pp. 303-348). New York: Academic Press.

NoBlE, W. (1981). Earmuffs, exploratory head movements, and horizontal and vertical sound localization. Journal of Auditory Research, 21, 1-12.

Noble, W., Byrne, D., \& LePage, B. (1994). Effects on sound localization of configuration and type of hearing impairment. Journal of the Acoustical Society of America, 95, 992-1005.

Noble, W., Murray, N., \& WaUgh, R. (1990). The effect of various hearing protectors on sound localization in the horizontal and vertical planes. American Industrial Hygiene Association Journal, 51 , 370-377.

OldField, S., \& PARKer, S. (1984). Acuity of sound localisation: A topography of auditory space: I. Normal hearing conditions. Perception, 13, 581-600. 
Searle, C. L., Braida, L. D., Cuddy, D. R., \& Davis, M. F. (1975) Binaural pinna disparity: Another auditory localization cue. Journal of the Acoustical Society of America, 57, 448-455.

Searle, C. L., Braida, L. D., Davis, M. F., \& Colburn, H. S. (1976). Model for auditory localization. Journal of the Acoustical Society of America, 60, 1164-1175.

SHAW, E. A. G. (1982). External ear response and sound localization In R. W. Gatehouse (Ed.), Localization of sound: Theory and applications (pp. 30-41). Groton, CT: Amphora.

Stevens, S. S., \& Newman, E. B. (1936). The localization of actual sources of sound. American Journal of Psychology, 48, 297-306.

Thurlow, W. R., \& RUNGE, P. S. (1967). Effect of induced head movements on localization of direction of sounds. Journal of the Acoustical Society of America, 42, 480-488.
WightMan, F. L., \& Kistler, D. J. (1989). Headphone simulation of free-field listening: II. Psychophysical validation. Journal of the Acoustical Society of America, 85, 868-878.

Wightman, F. L., \& Kistler, D. J. (1993). Sound localization. In R. Fay, A. Popper, \& W. Yost (Eds.), Springer series in auditory research: Human psychophysics (pp. 155-192). New York: SpringerVerlag.

WOODWORTH, R. S. (1938). Experimental psychology. New York: Holt, Rinehart \& Winston.

(Manuscript received April 1, 1994; revision accepted for publication September 3, 1994.) 